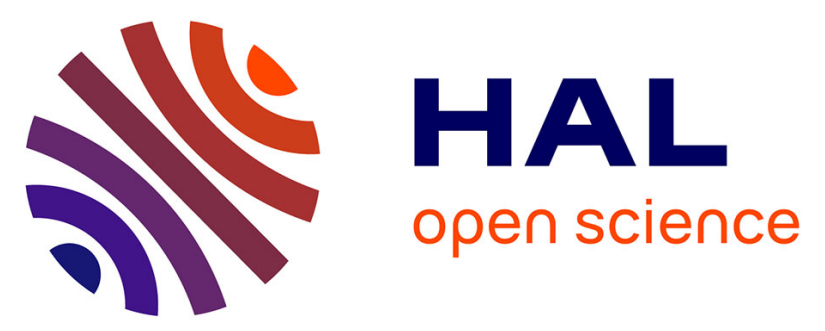

\title{
Upscaling permeability for fractured concrete: meso-macro numerical approach coupled to strong discontinuities
}

Xavier Jourdain, Jean-Baptiste Colliat, Caroline de Sa, Farid Benboudjema, Fabrice Gatuingt

\section{To cite this version:}

Xavier Jourdain, Jean-Baptiste Colliat, Caroline de Sa, Farid Benboudjema, Fabrice Gatuingt. Upscaling permeability for fractured concrete: meso-macro numerical approach coupled to strong discontinuities. International Journal for Numerical and Analytical Methods in Geomechanics, 2014, 38 (5), pp.536-550. 10.1002/nag.2223 . hal-01006624

\section{HAL Id: hal-01006624 \\ https://hal.science/hal-01006624}

Submitted on 16 Jun 2014

HAL is a multi-disciplinary open access archive for the deposit and dissemination of scientific research documents, whether they are published or not. The documents may come from teaching and research institutions in France or abroad, or from public or private research centers.
L'archive ouverte pluridisciplinaire HAL, est destinée au dépôt et à la diffusion de documents scientifiques de niveau recherche, publiés ou non, émanant des établissements d'enseignement et de recherche français ou étrangers, des laboratoires publics ou privés. 


\title{
Upscaling permeability for fractured concrete: meso-macro numerical approach coupled to strong discontinuities
}

\author{
X. Jourdain*, J.-B. Colliat**, C. De Sa*, F. Benboudjema* and F. Gatuingt* \\ *LMT-Cachan (ENS Cachan/CNRS/Université Paris 6/PRES UniverSud Paris), France \\ **LML, Univ. Lille 1, CNRS, Ecole Centrale de Lille, Arts et Métiers ParisTech, France
}

\begin{abstract}
A two scales numerical analysis is set up in order to upscale the permeability of fractured materials such as concrete. To that aim we couple FE kinematics enhancements (strong discontinuities) representing fine scale cracks to the fine scale permeability tensor. The latter may be split into two parts: the first one is isotropic and corresponds to flows within the porosity of the material; the second one, based upon a set of cracks with different orientations and openings, is anisotropic. For the latter each crack is a path for mass flow according to the Poiseuille law considering two infinite planes. We show how the upscaling procedure leads both to the definition of macroscopic permeability tensors as well as the flow rate evaluation for components of concrete structures.
\end{abstract}

\section{Introduction}

Mass (gas and water) transfers within concrete are an important issue dealing with numerous applications in Civil engineering such as geological $\mathrm{CO}_{2}$ storage or civil nuclear industry. In France, the latter aims at extending its nuclear powerplants lifetime which requires accurate quantifications of the outflow through the concrete vessel along different cases and accounting for the long-term evolution of concrete properties and cracking. Under severe accident cases - e.g. LOCA: Loss-Of-Coolant Accident - , the stress and strain states may reach quite important values, leading to a set of macroscopic cracks. The main objective is then to assess that the leaking rate remains below some prescribed threshold, which is usually done thanks to some integrity tests.

On the physical point of view, macroscopic cracks lead to a macroscopic permeability that increases by several orders of magnitude and also exhibits a strong anisotropy due to cracks orientations [1], [2]. Except for low stress levels, linking the fractured state of concrete to the induced anisotropic permeability tensor is a difficult task [3], [4]. The main reason for that is the broad use of macroscopic smeared crack models which are not providing accurate and physical quantities - such as cracks opening and orientation - in order to model fractures within a FE context. Actually, it is quite clear that the "discrete" nature of each macroscopic fracture - and thus of the corresponding flow - also requires a discrete numerical representation of cracks in order to set up the hydro-mechanical coupling on a physical basis. Such bridge have been made using, for example, lattice models [5]. On the other hand, considering a set of meso-scale cracks with different openings and orientations, the problem of determining the macroscopic permeability can also be handled in an analytical sense through self-consistent scheme [6]. The latter shows that the order of magnitude of the permeability 
increases beyond a meso-scale crack density threshold. However, such analytical scheme requires to assume some constraint about the cracks distribution (e.g. isotropic orientations) and is thus mostly restricted to low levels or isotropic loading cases. Here we show that incorporating discontinuities which are, by essence, discrete — within the FE interpolation of the displacements field also leads to a natural and accurate way to quantify both macroscopic flows and permeability tensors.

To that aim we consider a two scales approach, in the spirit of sequential multi-scale methods [7]. The latter tend to build macroscopic models from a sequence of lower scale computations. Most of them rely on a probabilistic description of phases arrangement or material properties at the fine scale. Here they require both a macroscale flow model — chosen among porous media flow theory — and a fine scale coupled hydromechanical analysis. By applying a set of specific boundary conditions at the mesoscale - fine scale — and assuming ergodicity, the spatial averages allow to compute macroscopic quantities, such as permeability tensors according to the chosen macroscopic flow model. This a posteriori upscaling makes sequential multi-scale methods quite popular in order to compute macroscopic properties, especially considering non-linear behavior for which analytical homogenization meets important theoretical challenges. It is worth noting that, whether ergodicity doesn't appear as a correct assumption (eg. dealing with non differentiable in time processes), the methodology is still valid, provided that the spatial averages are replaced by ensemble integrals. The latter may be advantageously computed through Monte-Carlo integration. The latter basically consists in considering several independent mesoscale realizations and in computing a posteriori statistics.

The outline of this paper is as follows: the sequential multi-scale framework is detailed in Section 2, as well as the cracks FE modeling and the hydro-mechanical coupling, both at mesoscale. In Section 3 we next turn to the mesoscale flow problem statement, considering both incompressible and compressible flows. Section 4 shows the results of the upscaling procedure, first dealing with different "idealized" cracks patterns. Finally we consider a Reinforced Concrete element submitted to tension and compare the numerical simulations to the main experimental results from [8].

\section{Sequential multi-scale framework}

As opposed to integrated multi-scale methods, sequential ones require both a macroscopic model and a fine scale one. The former defines a set of macroscopic parameters which may be identified thanks to a sequence of fine scale computations along specific boundary conditions and spatial averaging. Here the macroscopic point of view is that mass transfers through concrete can be modeled in the sense of porous media flow [9], which defines the macroscopic permeability as a symmetric and positive-definite second order tensor $\underline{\underline{\mathbf{K}}}^{\mathrm{M}}\left[\mathrm{m}^{2}\right]$ according to,

$$
\underline{\mathrm{Q}}^{\mathrm{M}}=-\frac{\rho}{\mu} \underline{\underline{\mathbf{K}}}^{\mathrm{M}} \cdot \underline{\mathrm{G}}^{\mathrm{M}}\left[\mathrm{kg} \cdot \mathrm{s}^{-1} \cdot \mathrm{m}^{-2}\right]
$$

where $\mu$ and $\rho$ are the viscosity and the density of the fluid. $\underline{Q}^{\mathrm{M}}$ is the macroscopic mass flow density vector and $\underline{G}^{\mathrm{M}}$ is the macroscopic pressure gradient, both defined as spatial averages of the corresponding fine scale quantities:

$$
\underline{\mathrm{Q}}^{\mathrm{M}}=\frac{1}{V} \int_{V} \underline{\mathrm{q}}(\underline{\mathrm{x}}) \mathrm{dV}, \underline{\mathrm{G}}^{\mathrm{M}}=\frac{1}{V} \int_{V} \underline{\operatorname{grad}}(p) \mathrm{dV}
$$

In order to compute each components of the tensor $\underline{\mathbf{K}}^{\mathrm{M}}$, a sequence of mesoscale computations have to be performed along specific boundary conditions which must be chosen according to the 
Hill-Mandel condition [10]. Such condition ensures that the dissipations at small and large scales are matching (in a statistical sense) and thus allows to evaluate the macroscopic constitutive response of heterogeneous media in terms of spatial averages. It has been reformulated in the case of porous media flow by [11] leading us to stress on two kinds of uniform boundary conditions which are,

- the uniform pressure boundary condition (Dirichlet-type) defined as,

$$
p(\underline{\mathrm{x}})=\underline{\mathrm{G}}^{\mathrm{M}} \cdot \underline{\mathrm{x}}+p_{0} \quad \forall \underline{\mathrm{x}} \in \partial \Omega
$$

where $\underline{G}^{\mathrm{M}}$ is the pressure gradient which is, in this case, considered to be a constant vector. To be more precise, considering $\underline{G}^{\mathrm{M}}=\left[\begin{array}{lll}1 & 0 & 0\end{array}\right]^{T}$ and the two other circular permutations lead to a straightforward way to compute the nine components of the macroscale permeability matrix $\underline{\underline{\mathrm{K}}}^{\mathrm{M}}$.

- the uniform mass flow boundary condition (Neumann-type) as,

$$
\underline{\mathrm{q}}(\underline{\mathrm{x}}) \cdot \underline{\mathrm{n}}(\underline{\mathrm{x}})=\rho(\underline{\mathrm{x}}) \underline{\mathrm{v}}(\underline{\mathrm{x}}) \cdot \underline{\mathrm{n}}(\underline{\mathrm{x}})=\underline{\mathrm{Q}}^{\mathrm{M}} \cdot \underline{\mathrm{n}}(\underline{\mathrm{x}}) \quad \forall \underline{\mathrm{x}} \in \partial \Omega
$$

where $\underline{\mathrm{Q}}^{\mathrm{M}}$ is a constant vector, $\underline{\mathrm{n}}(\underline{\mathrm{x}})$ is the unit normal vector and $\underline{\mathrm{v}}$ is the filtration velocity. Considering $\underline{Q}^{\mathrm{M}}=\left[\begin{array}{lll}1 & 0 & 0\end{array}\right]^{T}$ and the two other circular permutations allows for a complete definition of $\left(\underline{\underline{\mathbf{K}}}^{\mathrm{M}}\right)^{-1}$.

The influence of the choice for the boundary conditions is discussed at the beginning of Section 4 . However, whether the two results are diverging, we shall already stress on the ability of the uniform pressure case to provide more reliable results compared to analytical solutions.

Such two-scale framework thus requires to set up both a macro model (to be identified) and a mesoscale one. Here, following [12] the latter is based on a spatial truss representation in order to model the mechanical behavior of a two phases material (aggregates melt into mortar). The kinematics enhancements through a set of strain discontinuities lead to FE meshes which are not constrained by the physical interfaces, hence allowing for an accurate and simple way to handle complex mesoscale geometries [13]. On the other hand, the non-linear behaviour is captured by meso-scale cracks modeling through a set of displacement discontinuities.

\subsection{Mesoscale failure FE modeling: embedded strong discontinuity}

One of the key issues dealing with the numerical modeling of brittle and quasi-brittle materials is the fracture process modeling and its representation. From a physical point of view, fractures or cracks might be viewed as introducing displacement discontinuities within a continuum. For modeling aspects, cracking leads to the progressive decrease of the stiffness and eventually to softening behavior where strain increase is accompanied by the stress decrease. It is well-known that dealing with such kind of softening behavior leads to non-uniqueness of $\mathrm{FE}$ solutions with respect to the chosen mesh. Several numerical techniques have been developed during the past twenty years in order to regularize FE models including softening laws. Among them, it is worth citing the smeared crack model [14], the non-local approach [15] and more recently the strong discontinuity approach - [16], [17], [18]. The latter has proven to be quite efficient without any requirement in terms of additional parameter and is widely used in order to model continuum material failure. Two alternatives, nodal (X-FEM [18]) and element (E-FEM [16]) enrichments, are presently available for capturing displacement (strong) discontinuities. Deciding which one is the most suitable for the purpose of material failure simulation 

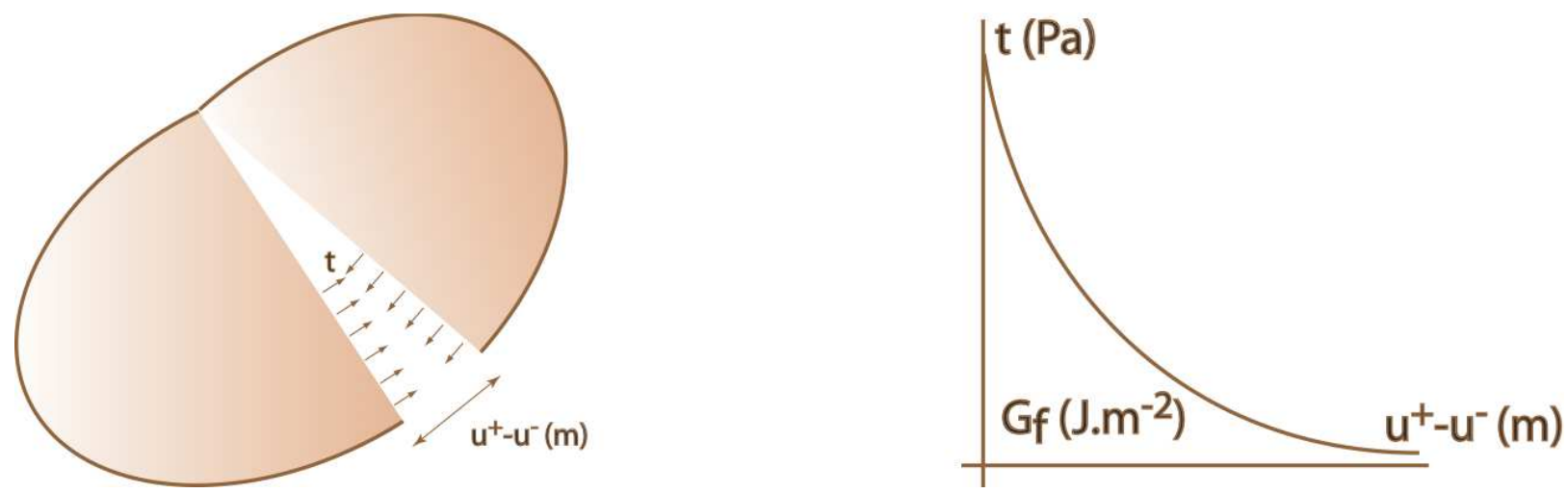

Figure 1: Strong discontinuity crack representation and crack opening process

is still a controversial issue, out of scope of this work. The numerical simulations presented in this work have been carried out using element enrichments, which benefit from the static condensation procedure. The main advantage of such a methodology is to be able to provide numerical values for cracks opening and to preserve the total number of unknowns as well. Adding strong enhancements to the set of strain discontinuities thus lead to a non-linear modeling strategy which is oriented towards a more reliable interpretation of failure mechanisms for heterogeneous materials.

Here, within the context of the E-FEM methods, the mechanical analysis is based on [12] and [19], and relies on spatial truss models at meso-scale. Such truss is generated by first computing the 3D Delaunay triangulation of a Poisson point process over the spatial domain of interest in accordance with a prescribed points density. Each edge of the resulting tetrahedral elements is then converted into a bar element whose cross-section is computed by mean of the corresponding Voronoi tessellation. Finally, spherical inclusions are generated from the Gibbs point process, taking into account both prescribed volume fraction and inclusion diameters.

Moreover, dealing with brittle and quasi-brittle materials, pretty simple failure mechanisms are introduced at meso-scale. The chosen yield function $\Phi$, which leads to activating the strong discontinuities within the elements, corresponds to tensile fracture and mode I opening. Here it is written as,

$$
\Phi(t, q)=t-\left(\sigma_{f}-q\right) \leq 0
$$

where $t$ is the traction vector at the discontinuity and $\sigma_{f}$ the limit stress. The softening is introduced through the variable $q=f(\llbracket u \rrbracket)$ by considering the exponential form,

$$
f(\llbracket u \rrbracket)=\sigma_{f}\left(1-\exp \left(-\frac{\sigma_{f}}{G_{f}} \llbracket u \rrbracket\right)\right)
$$

where $\llbracket u \rrbracket=u^{+}-u^{-}$is the displacement jump — which is assumed to be the image of the crack opening and belongs to the set of unknowns - and the fracture energy Gf [J.m $\left.{ }^{-2}\right]$ is introduced as the area under the $t$ vs. $\llbracket u \rrbracket$ curve (see Fig.1). It is worth noting that such fine scale failure criterion is triggered only for fracture in tension (or equivalently in case of extension dealing with truss elements) at meso-scale, which is typical of brittle or quasi-brittle materials like cement-based ones. However, [12] showed that the macroscopic mechanical response of such models exhibits failure modes along several macroscopic proportional loading paths, such as simple tension and compression, shear, bi-axial tension and compression, etc. Moreover, here the key point are the embedded displacement 
discontinuities which are assumed to model meso-scale cracks through a finite jump within the displacement interpolation. The magnitude $\llbracket u \rrbracket$ of this jump is nothing else but the crack opening. It is quite clear that such an information leads to a straightforward way to compute mass flows within meso-scale cracks. Moreover the set of cracks orientations clearly gather the whole information dealing with the fracture pattern anisotropy and thus allows to set up an accurate hydro-mechanical coupling at such fine scale by the mean of Finite Element assembly procedure. This point is detailed in the next section.

\subsection{Meso-scale coupling through the Poiseuille law}

The meso-scale mechanical representation developed in [12] is based on a spatial truss built thanks to a Poisson point process [20] and its Delaunay triangulation. The latter defines a set of two nodes finite elements, which kinematics are enhanced in order to represent both heterogeneities and fine scale cracks. Here we assume that each of those crack is a path for a mass flow, according to the Poiseuille law written in case of two infinite parallel planes. Obviously, the distance between those planes is equal to the meso-crack opening or displacement jump $w=\llbracket u \rrbracket=u^{+}-u^{-}$.

Dealing with an incompressible fluid, any meso-scale crack — which unit normal vector is $\underline{\mathrm{n}}$ leads to an equivalent fine scale porous media, with permeability tensor as,

$$
\underline{\underline{\mathbf{K}}}^{\mathrm{m}}=k_{i n t r} \underline{\underline{\mathbf{1}}}+\frac{w^{3}}{12 L}(\underline{\underline{\mathbf{1}}}-\underline{\mathrm{n}} \otimes \underline{\mathrm{n}})
$$

where $L$ is the length of a typical element including a strong discontinuity of magnitude $w$ and $k_{\text {intr }}$ is the intrinsic permeability of the material depending upon the porosity and the saturation degree (e.g. $10^{-17} \mathrm{~m}^{2}$ for ordinary concrete). It is worth noting that $k_{\text {intr }}$ must be strictly positive in order to get a full rank matrix $\underline{\underline{\mathbf{K}}}^{\mathrm{m}}$. The corresponding mass flow density vector $\underline{\mathrm{q}}$, due to such meso-scale crack, can thus be written as:

$$
\underline{\mathrm{q}}=-\rho \frac{\underline{\underline{\mathbf{K}}}^{\mathrm{m}}}{\mu} \cdot \underline{\operatorname{grad}}(p)
$$

This mass flow vector is clearly oriented along the crack and the latter has no influence in the normal direction:

$$
\underline{\mathrm{q}} \cdot \underline{\mathrm{n}}=-\rho \frac{k_{\text {intr }}}{\mu} \underline{\operatorname{grad}}(p) \cdot \underline{\mathrm{n}}
$$

Considering a compressible fluid, here assumed to follow the ideal gas law,

$$
\rho=p \frac{M}{R T}
$$

where $M$ is its molar mass $\left(\mathrm{kg} \cdot \mathrm{mol}^{-1}\right), R$ is the universal gas constant $\left(8,314 \mathrm{~J} \cdot \mathrm{K}^{-1} \cdot \mathrm{mol}^{-1}\right)$ and $T$ the absolute temperature $(\mathrm{K})$, the density cannot be assumed to remain constant and (8) must be reformulated as,

$$
\underline{\mathrm{q}}=-\frac{\underline{\underline{\mathbf{K}^{\mathrm{m}}}}}{\mu} \frac{M}{R T} p \cdot \underline{\operatorname{grad}}(p)
$$

or, in a more practical way as:

$$
\underline{\mathrm{q}}=-\underline{\underline{\underline{\mathbf{K}}}}_{2 \mu}^{\mathrm{m}} \frac{M}{R T} \cdot \underline{\operatorname{grad}}\left(p^{2}\right)
$$

Equation (12) is very similar to (8), apart from showing a linear relationship between the mass flow density and the gradient of the pressure-squared field and not the pressure field itself. This 
remark suggests a common resolution scheme for both compressible and incompressible flows (see next section).

Having this fine scale hydro-mechanical coupling in hands, it is clear that any mass flow problem shall inherit from most of the information coming from the mechanical meso-scale analysis. The corresponding set of meso-scale cracks, each showing different openings and orientations, shall lead to a straightforward way to account for both induced anisotropy, fracture tortuosity and percolation issue. In order to set up such information upscaling, we here rely on a FE formulation of the flow problem at meso-scale. Hence, by defining an elementary permeability matrix for each element and by applying the FE assembly procedure, the resolution of this problem leads to a complete definition of the flow solution. The next section focuses on the FE formulation of this flow problem.

\section{FE formulation of the global flow problem}

The keystone of any sequential multi-scale analysis is obviously the ability to numerically solve physical problems at the chosen "fine" scale. Here, we focus on porous media flow problems within the context of the hydro-mechanical coupling presented above. As stated previously, the latter leads to a heterogeneous field of anisotropic permeability tensors. Moreover we aim at gathering within a common numerical scheme both compressible and incompressible flows. Since the latter is extensively described in numerous textbooks [9], [21], here we focus on compressible fluids, for which using the pressure-squared field leads to such a common FE simulation scheme. We start by recalling the weak form of the mass balance equation, before turning to the FE spatial discretization. Finally we provide some details dealing with the elementary matrix computation.

\subsection{Weak form of the mass balance equation}

Assuming steady-state flows and no source, the local — strong — form of the mass balance equation is,

$$
\operatorname{div}(\rho \underline{\mathrm{v}})=\operatorname{div}(\underline{\mathrm{q}})=0
$$

where $\underline{q}$ is still the mass flow density vector $\left[\mathrm{kg} \cdot \mathrm{s}^{-1} \cdot \mathrm{m}^{-2}\right]$. Such form - substituting mass flow to filtration velocity - is suitable for both incompressible and compressible cases. The corresponding weak form can be written as follows:

Find $p^{2} \in S(\Omega)$, such that $\forall p^{\star 2} \in V(\Omega)$ :

$$
\int_{\Omega} p^{\star 2} \operatorname{div}\left(\underline{\mathrm{q}}\left(p^{2}\right)\right) \mathrm{d} \Omega=0
$$

Here $S(\Omega)$ is the admissible pressure-squared field space (an affine space),

$$
S(\Omega)=\left\{p^{2} \in H^{1}(\Omega) \mid p^{2}(\partial \Omega)=p_{0}^{2}\right\}
$$

where $H^{1}(\Omega)=\left\{p^{2} \in L^{2}(\Omega) ; \forall i=1 \ldots n, \frac{\partial p^{2}}{\partial x_{i}} \in L^{2}(\Omega)\right\}$ is a Hilbert space. $V(\Omega)$ is the admissible pressure-squared fields space to zero (i.e. nil on the part of the boundary for which Dirichlet conditions are applied):

$$
V(\Omega)=\left\{p^{\star 2} \in H^{1}(\Omega) \mid p^{\star 2}(\partial \Omega)=0\right\}
$$


With both an integration by parts and the Green-Ostrogradski theorem, equation (14) becomes,

$$
\int_{\delta \Omega_{q}} p^{\star 2} \underline{\mathrm{q}}_{0} \cdot \underline{\mathrm{n}} \mathrm{d} \mathrm{s}-\int_{\Omega} \underline{\operatorname{grad}}\left(p^{\star 2}\right) \cdot \underline{\mathrm{q}} \mathrm{d} \Omega=0
$$

which is the basis to compute an approximate solution $p^{2, h}$ of the flow problem.

\subsection{Approximate FE solution}

A solution to (17) is seeked within a finite dimensional subspace $S^{h}\left(\Omega^{h}\right) \subset S(\Omega)$. Providing a finite element mesh $\Omega^{h}=\bigcup_{e=1}^{n_{e l}} \Omega^{e}$ of the domain, an ansatz function $N_{a}(\underline{\mathrm{x}})$ is associated to each node of this mesh. The whole set of these ansatz functions span the finite-dimensional space $S^{h}\left(\Omega^{h}\right)$ of which they form a basis. Over a finite element $\Omega_{e}$, the approximate solution $p^{2, h}$ can thus be written as,

$$
\left.p^{2, h}(\underline{\mathrm{x}})\right|_{\Omega_{e}}=\sum_{a} N_{a}(\underline{\mathrm{x}}) p_{a}^{2}=\underline{\mathrm{N}} \cdot \underline{\mathrm{p}}_{e}^{2}
$$

where the vector $\underline{\mathrm{p}}_{e}^{2}$ contains the pressure-squared values on each node of $\Omega_{e}$. Gathering the derivatives of the ansatz functions within elementary matrices $\mathrm{B}_{e}$, we may write the gradient of the pressuresquared field as,

$$
\left.\underline{\operatorname{grad}}^{h}\left(p^{2}\right)\right|_{\Omega_{e}}=\mathrm{B}_{e} \cdot \underline{\mathrm{p}}_{e}^{2}
$$

With the above definitions and the approximate weak form on $\Omega^{h}$ coming from (17), we obtain a set of equations,

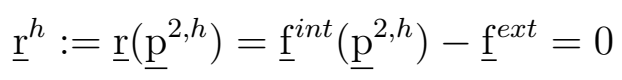

where

$$
\underline{\underline{i}}^{i n t}=-\AA_{e=1}^{n_{e l}} \int_{\Omega_{e}} \mathrm{~B}_{e}^{\mathrm{T}} \cdot \underline{\mathrm{q}} \mathrm{d} \Omega
$$

is the internal forces vector and $\mathrm{A}_{e=1}^{n_{e l}}$ is the finite element assembly operator. Still dealing with compressible flows, (12) provides a linear relationship between the mass flow density vector q and the pressure-squared field $p^{2}$. Including this relation in (20) leads to a set of linear equations to be solved,

$$
\mathcal{K} \cdot \underline{\mathrm{p}}^{2, h}=\underline{\mathrm{f}}^{e x t}
$$

where $\mathcal{K}$ is the FE permeability matrix associated to the chosen mesh $\Omega^{h}$,

$$
\mathcal{K}=\AA_{e=1}^{n_{e l}} \mathcal{K}^{e}
$$

and $\mathcal{K}^{e}$ are the elementary permeability matrix:

$$
\mathcal{K}^{e}=\int_{\Omega_{e}} \mathrm{~B}_{e}^{\mathrm{T}} \cdot \frac{M}{R T} \frac{\underline{\underline{\mathbf{K}}}^{\mathrm{m}}}{\overline{2 \mu}} \cdot \mathrm{B}_{e} \mathrm{~d} \Omega
$$




\subsection{Elementary permeability matrix}

Considering (7), the elementary permeability matrix defined by (24) can obviously be split into two parts,

$$
\mathcal{K}^{e}=\mathcal{K}_{\text {iso }}^{e}+\mathcal{K}_{\text {aniso }}^{e}
$$

where $\mathcal{K}_{i s o}^{e}$ is the isotropic part corresponding to the uncracked material,

$$
\mathcal{K}_{i s o}^{e}=\int_{\Omega_{e}} \mathrm{~B}_{e}^{\mathrm{T}} \frac{M}{R T} \frac{k_{\text {intr }}}{2 \mu} \cdot \mathrm{B}_{e} \mathrm{~d} \Omega
$$

and $\mathcal{K}_{\text {aniso }}^{e}$ is the anisotropic part linked to mesoscale failures:

$$
\mathcal{K}_{\text {aniso }}^{e}=\int_{\Omega_{e}} \mathrm{~B}_{e}^{\mathrm{T}} \cdot \frac{M}{\mu R T} \frac{w^{3}}{24 L}(\underline{\underline{\mathbf{1}}}-\underline{\mathrm{n}} \otimes \underline{\mathrm{n}}) \cdot \mathrm{B}_{e} \mathrm{~d} \Omega
$$
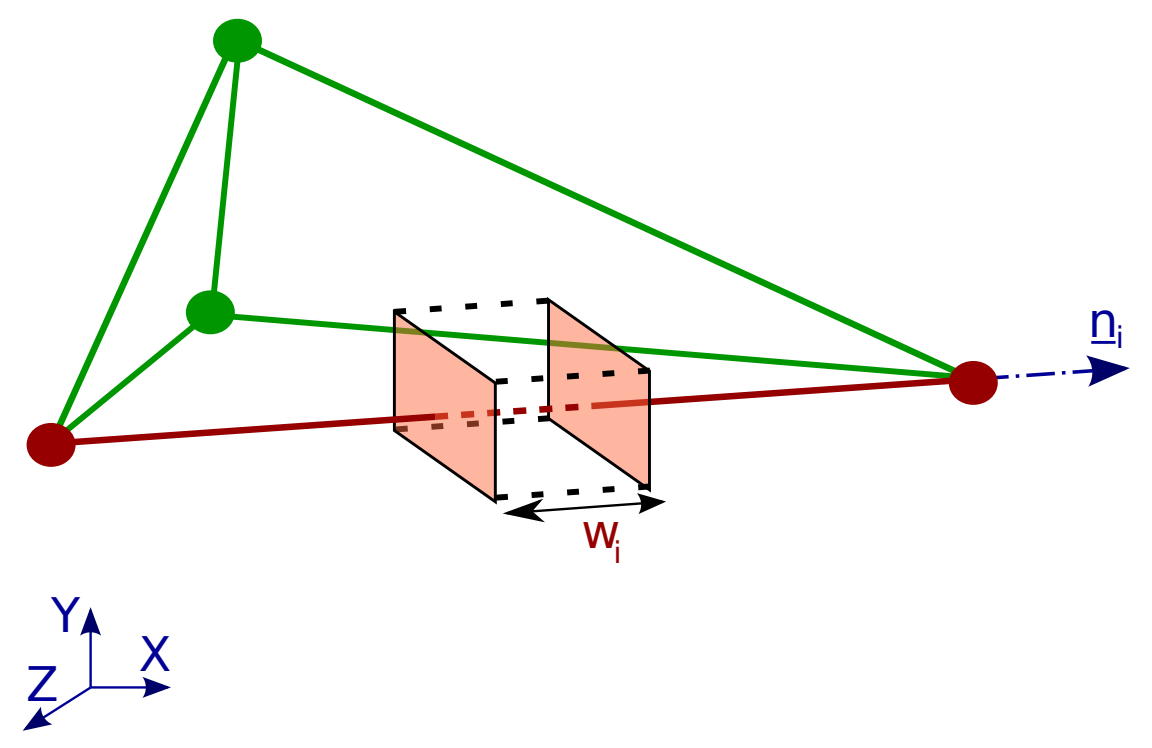

Figure 2: 4-nodes tetrahedron flow element with one mesoscale crack

Equation (27) states for one mesoscale crack — oriented along $\underline{n}$ — only. Here we deal with 4-nodes tetrahedron element which thus consist of six edges. Hence, each of those edges represents an element within the mechanical fine scale lattice (see Fig.2 where one flow analysis element is represented - a 4-nodes tetrahedron - with one strong discontinuity) and thus may be associated to a fine scale crack whose opening is computed numerically. Consequently, the anisotropic part of (25) has to be written as,

$$
\mathcal{K}_{\text {aniso }}^{e}=\sum_{i=1}^{6} \int_{\Omega_{e}} \mathrm{~B}_{e}^{\mathrm{T}} \cdot \frac{M}{\mu R T} \frac{w_{i}^{3}}{24 L}\left(\underline{\underline{\mathbf{1}}}-\underline{\mathrm{n}}_{i} \otimes \underline{\mathrm{n}}_{i}\right) \cdot \mathrm{B}_{e} \mathrm{~d} \Omega
$$

where the set of cracks associated to element $\Omega^{e}$ has $\underline{\mathrm{n}}_{i}$ orientations and $w_{i}$ openings. 
It is worth noting that, on the numerical procedure point of view, $\mathcal{K}_{i s o}^{e}$ evaluation requires a single numerical integration point. On the other hand, considering the set of six edges of $\Omega^{e}, \mathcal{K}_{\text {aniso }}^{e}$ evaluation can thus be viewed according to six numerical integration points.

Assembling the elementary permeability matrix (25) and solving the set of linear equations (22) leads to the approximate solution of the flow problem. This solution consists of the pressure field for incompressible fluid and of the pressure-squared field dealing with compressible ones. Both can thus be solved using the same FE code and procedures, and the only slight difference then remains in considering the pressure-squared for compressible flows.

With this two-scales numerical tool in hands we now turn to some specific numerical examples in order to demonstrate its main feature.

\section{Numerical examples and upscaling validation}

On the physical point of view, the macroscopic permeability tensor must clearly inherits from the anisotropy due to the crack patterns. The numerical examples presented in this section show that the upscaling process leads to such result thanks to the Finite Element assembly procedure. To that aim, we first consider "perfect" failure patterns — which consist of a single plane crack with constant opening from $10^{-6} \mathrm{~mm}$ to $10^{-2} \mathrm{~mm}$ - inside a cubic domain. We compute the macroscopic permeability tensor along different orientations and opening values. Then we focus on reinforced concrete $(\mathrm{RC})$ element submitted to simple tension and for which a novel permeability device has been developped by [8].

On the Software Engineering point of view, those computations are based upon the FE simulator component coFeap [22] which allows for multiple instantiations and asynchronous communication. Hence the sequence of fine scale computations, which is the keystone of any sequential multi-scale method, may be performed in parallel using the same data set. Each of those computations consists in solving a compressible or incompressible flow problem using a fine mesh (here 338436 nodes and 1933643 tetrahedrons). Although mesh convergence studies (see Fig.3) show that an asymptotic response is obtained for much coarser grids, the chosen mesh for the flow problem must match with the spatial truss one. The latter's size is related to the diameter of the smallest inclusions to be modeled (here $1 \mathrm{~mm}$ ).

In order to solve the linear system (22) — or its counterpart dealing with incompressible flows we choose for wellknown iterative solver, like preconditioned conjugate gradient [23].

\section{1 "Perfect" failure patterns}

Figure 4 shows a single plane crack with constant opening range from $\llbracket u \rrbracket=10^{-6} \mathrm{~mm}$ to $\llbracket u \rrbracket=10^{-2}$ $\mathrm{mm}$. Apart from this specific path for mass flow, the whole domain $\left(100^{3} \mathrm{~mm}^{3}\right)$ is assumed to be homogeneous and with isotropic permeability $k_{\text {intr }}=10^{-17} \mathrm{~m}^{2}$. It is worth noting that such strictly positive value is mandatory in order to deal with full-rank permeability tensor (7).

Following the sequential multi-scale framework, a set of three different uniform pressure boundary conditions are imposed to the domain, leading to a complete determination of the macroscopic permeability tensor $\underline{\underline{\mathbf{K}}}^{\mathrm{M}}$. The three matrices (29), (30) and (31) show the evolution of this computed 


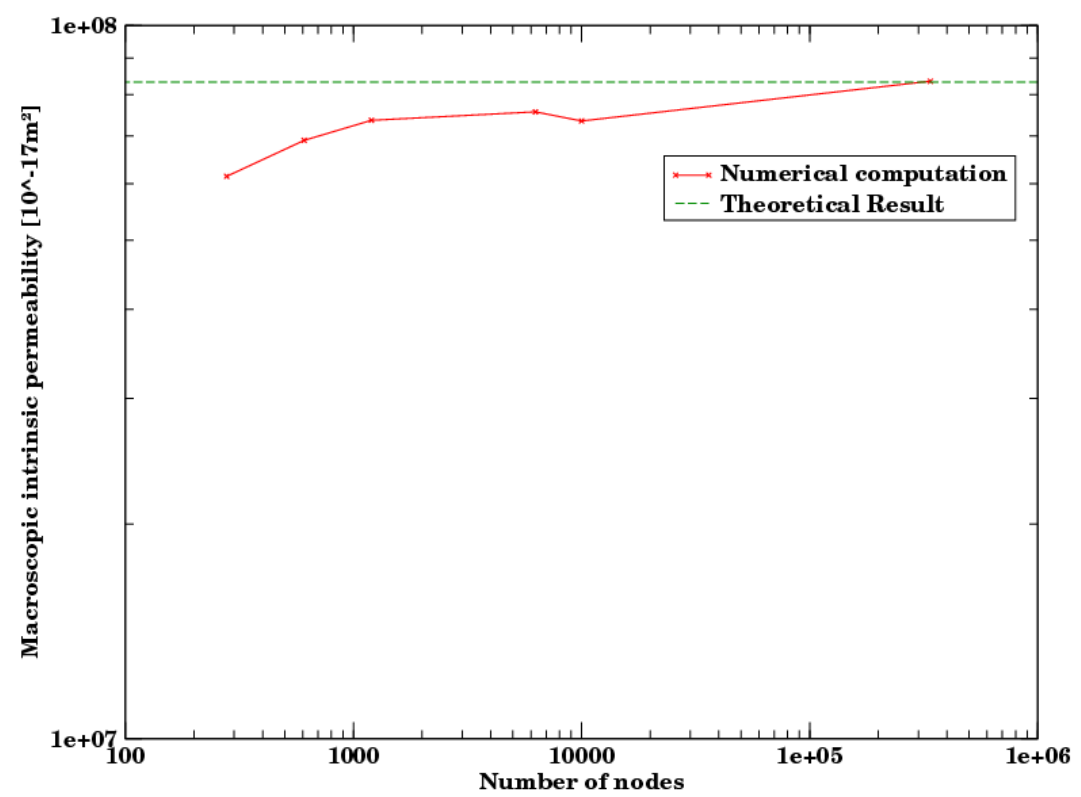

Figure 3: Mesh convergence considering a "perfect" plane crack — $1 \mathrm{~mm}$ opening — inside a $100^{3}$ $\mathrm{mm}^{3}$ domain

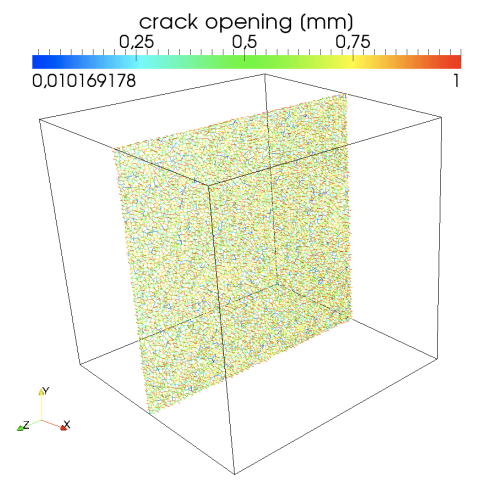

Figure 4: Single plane crack inside a cubic domain - the opening is constant in the normal direction $\mathrm{X}$

macroscopic tensor along the crack opening.

$$
\begin{gathered}
\underline{\underline{\mathbf{K}}}_{\llbracket u \rrbracket=10^{-6} \mathrm{~mm}}^{\mathrm{M}}=\left[\begin{array}{lll}
1.000 & 0.000 & 0.000 \\
0.000 & 1.000 & 0.000 \\
0.000 & 0.000 & 1.000
\end{array}\right] \cdot 10^{-17} \mathrm{~m}^{2} \\
\underline{\underline{\mathbf{K}}}_{\llbracket u \rrbracket=10^{-3} \mathrm{~mm}}^{\mathrm{M}}=\left[\begin{array}{ccc}
1.022 & 0.000 & 0.000 \\
0.000 & 1.170 & 0.000 \\
0.000 & 0.000 & 1.054
\end{array}\right] \cdot 10^{-17} \mathrm{~m}^{2} \\
\underline{\underline{\mathbf{K}}}_{\llbracket u \rrbracket=10^{-2} \mathrm{~mm}}^{\mathrm{M}}=\left[\begin{array}{ccc}
1.02 & 5.98 & 1.50 \\
-5.4 & 1.21 .10^{2} & 13.28 \\
2.08 & 18.10 & 1.07 .10^{2}
\end{array}\right] \cdot 10^{-17} \mathrm{~m}^{2}
\end{gathered}
$$

Clearly, (29) shows that a very tiny crack opening (comparing to the whole domain size) has no 
influence on the mass transfer properties. The macroscopic permeability tensor is then equal to the homogeneous meso-scale one. On the contrary, when dealing with larger openings, the influence of the crack becomes predominant. First, (31) shows a strong anisotropy which is clearly induced by the crack pattern (here a perfect plane). Second, the non diagonal components are no more equal to zero, especially considering the values that represents the cross-permeability within the plane defined by the crack. It is also worth noting that, although the above matrices are not strictly symmetric — which is mainly due to numerical precision dealing with iterative solvers - the norm of the nonsymmetric part of (31) is neglectable comparing to its symmetric part norm (less than $0.1 \%$ ).

Moreover, we stress here on the influence of the boundary conditions by comparing the two main cases offered by the Hill condition: "uniform pressure" or Dirichlet-type boundary conditions (3) and "uniform mass flow" or Neumann-type boundary conditions (4).

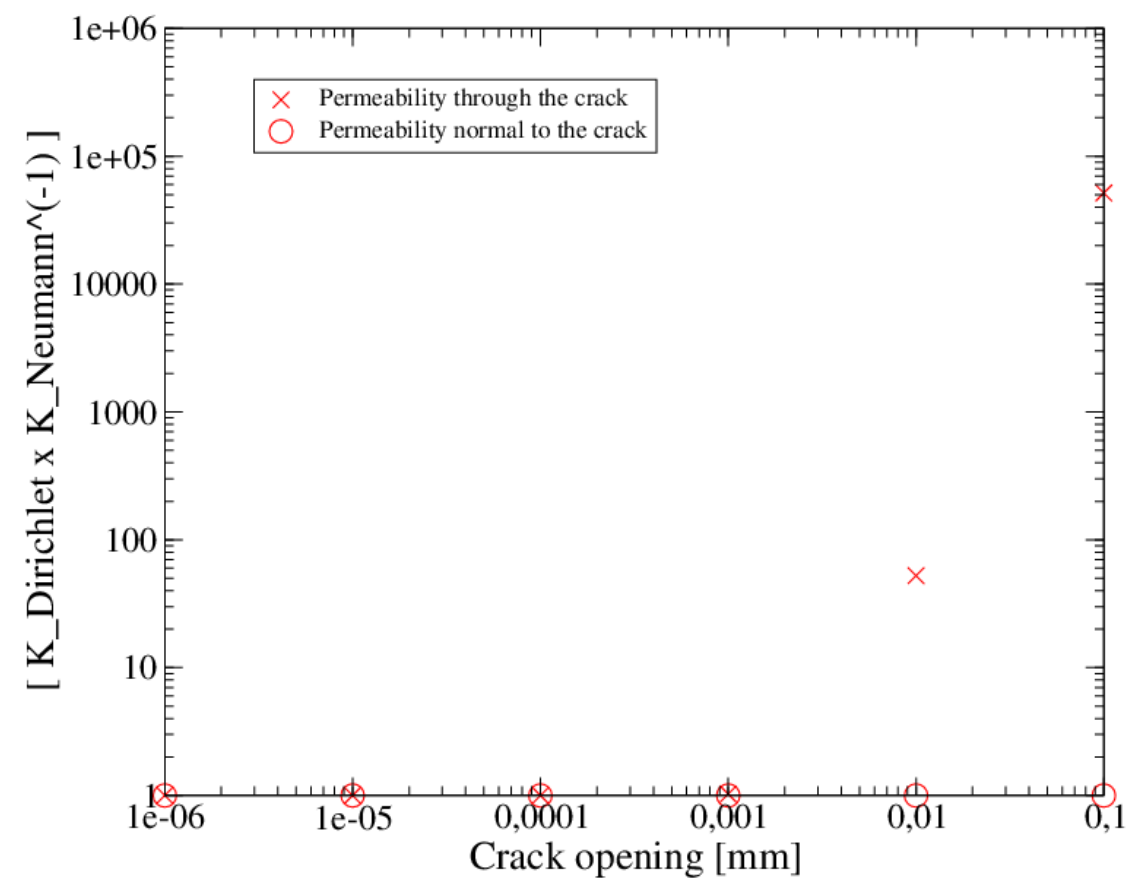

Figure 5: Influence of the boundary conditions : Comparison of Dirichlet and Neumann-type conditions

Figure 5 shows that this influence increases as the crack opening is growing as well. This is mainly due to the fact that, considering a single and oriented crack, the domain can be viewed as a Representative Volume Element provided the opening is very small only. When considering larger openings, the cube is not a RVE anymore, which leads to a major influence of the boundary conditions. In that case, Figure 6 shows that the uniform pressure choice is much more closer to the expected result - here the Poiseuille law — than the uniform mass flow one. Hence we choosed for the former in the subsequent results. This result is in accordance with the postulat formulated by Hill for mechanical problems [10]. He explains that the Representative Volume Element exists when the ratio of the mesoscopic length (length of the sample) over the microscale length (average size of the heterogeneity) "(a) is structurally entirely typical of the whole mixture on average, and (b) contains a sufficient number of inclusions of the apparent overall moduli to be effectively independent of the surface values of traction and displacement so long as these values are macroscopically uniform". In our study, as soon as the crack opens, the sample can't be seen as a RVE as it is "cut" in two parts 
which is in contradiction with the (a) proposition of Hill. The (b) proposition is also wrong as there is only one crack, which means one heterogeneity in the sample and therefore not a sufficient number of inclusions.

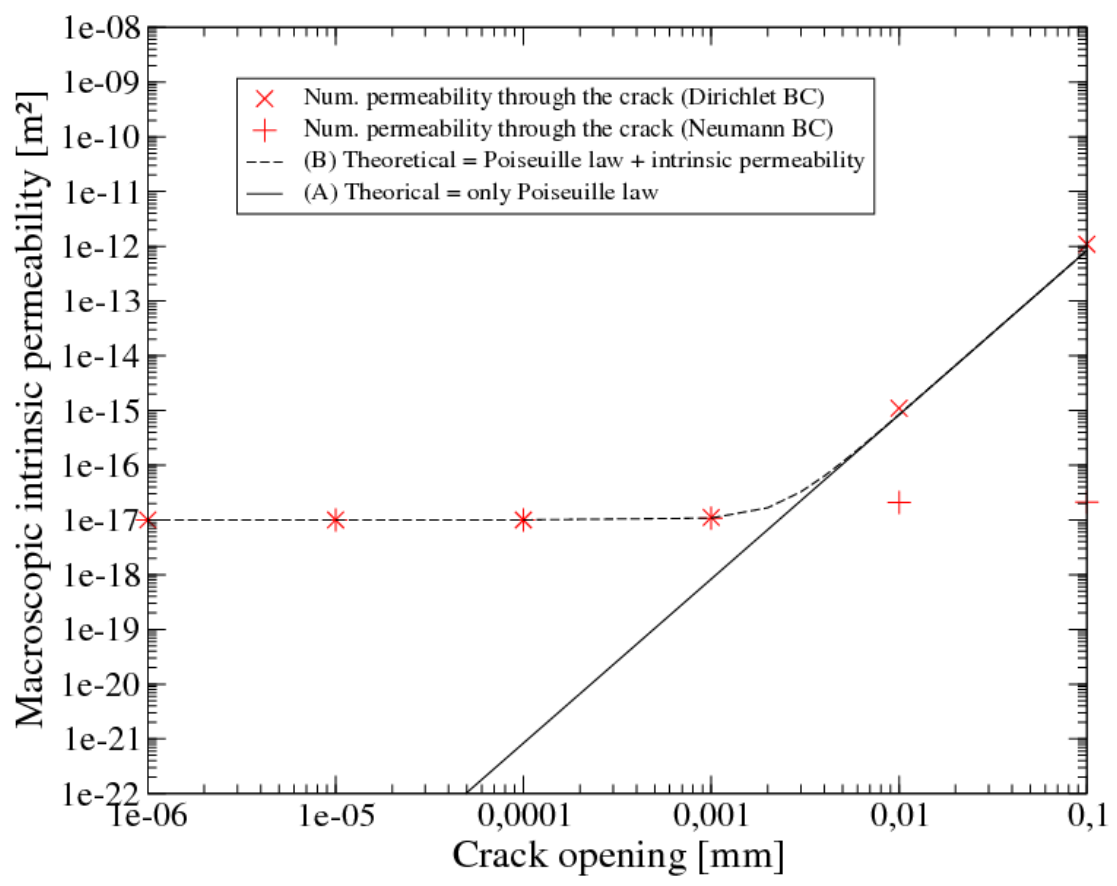

Figure 6: Influence of the boundary conditions : Comparison to analytical solution
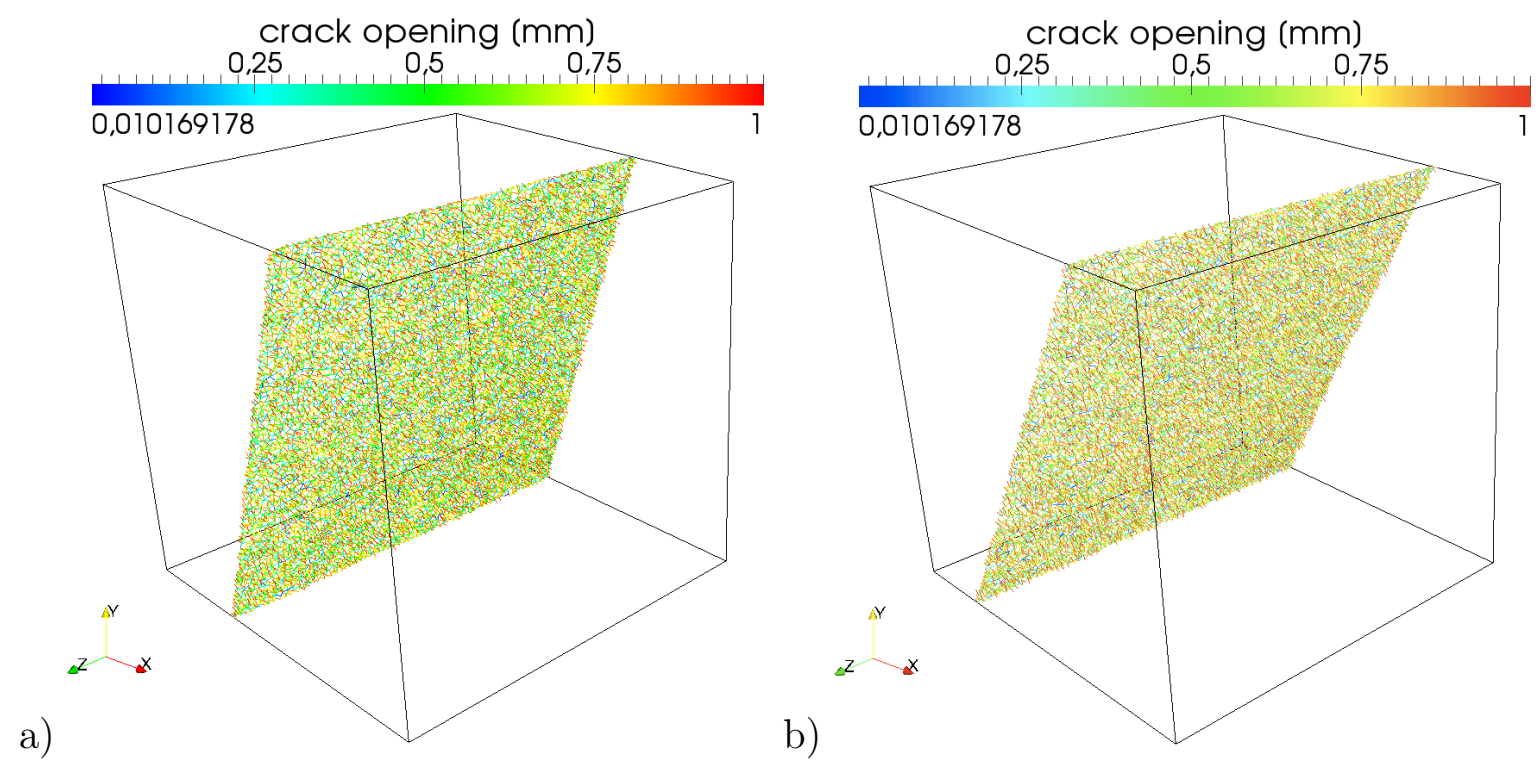

Figure 7: Plane cracks with $20^{\circ}$ and $30^{\circ}$ rotation around the $\mathrm{Z}$ axis

Figure 7 shows the same kind of "perfect" plane cracks, including a $20^{\circ}$ or $30^{\circ}$ rotation around 
the $\mathrm{Z}$ axis. The corresponding macroscopic permeability tensors are respectively,

$$
\underline{\underline{\mathbf{K}}}_{\left(\llbracket u \rrbracket=10^{-2} \mathrm{~mm}\right)}^{\mathrm{M}}=\left[\begin{array}{ccc}
12.1 & 26.5 & -0.03 \\
26.1 & 75.5 & -0.04 \\
-0.26 & -0.27 & 87.4
\end{array}\right] \cdot 10^{-17} \mathrm{~m}^{2}
$$

and

$$
\underline{\underline{\mathbf{K}}}_{\left(\llbracket u \rrbracket=10^{-2} \mathrm{~mm}\right)}^{\mathrm{M}}=\left[\begin{array}{ccc}
24.6 & 38.2 & 0.01 \\
37.6 & 68.8 & -0.01 \\
0.02 & 0.02 & 95.2
\end{array}\right] \cdot 10^{-17} \mathrm{~m}^{2}
$$

The smallest eigenvalue of the matrix (32) is $2.6 \times 10^{-17} \mathrm{~m}^{2}$ and $2.84 \times 10^{-17} \mathrm{~m}^{2}$ for matrix (33). The corresponding eigenvectors shows a $19.7^{\circ}$ angle for the first case and a $30.1^{\circ}$ angle for matrix (33), which can be compared to the $20^{\circ}$ and $30^{\circ}$ angles made by the crack planes. Moreover those values are in accordance with the intrinsic permeability of the homogeneous domain and thus shows that the crack has almost no influence on the mass transfer in the direction perpendicular to its plane. The largest two eigenvalues are $85.0 \times 10^{-17} \mathrm{~m}^{2}$ and $87.4 \times 10^{-17} \mathrm{~m}^{2}$ (dealing with matrix (32)) Those values are to be compared to the theoretical permeability corresponding to the Poiseuille law — with a $\llbracket u \rrbracket=1.10^{-2} \mathrm{~mm}$ opening — which is $83.3 \times 10^{-17} \mathrm{~m}^{2}$.

Finally, we compare the principal components of the macroscopic permeability tensor along the crack opening, from $\llbracket u \rrbracket=10^{-6} \mathrm{~mm}$ to $\llbracket u \rrbracket=10^{-2} \mathrm{~mm}$. Figure (8) shows that the computed tangent permeabilities follow the theoretical cubic law imposed by Poiseuille theory — introduced at the mesoscale - , while the normal components remains constant and almost equal to the intrinsic permeability of the surrounding domain.

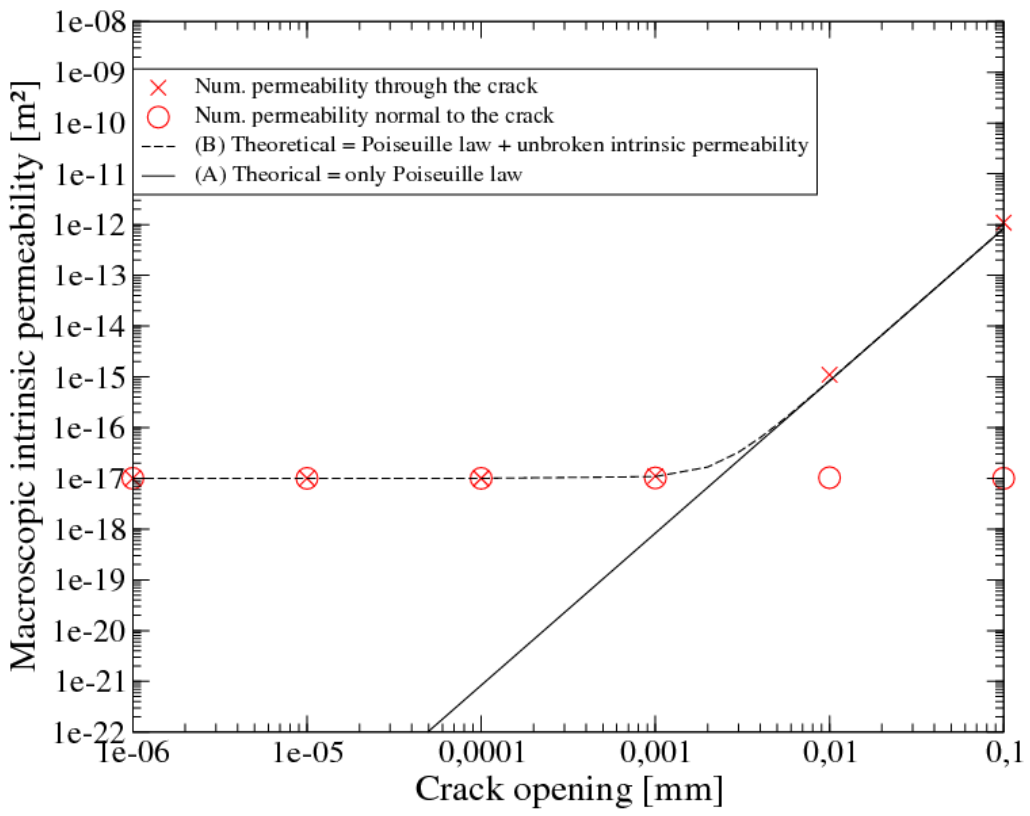

Figure 8: Evolution of the principal components of the macroscopic permeability tensor along the crack opening: ' $O$ ' stand for the normal direction and ' $x$ ' for the tangent directions

It is also worth noting that the influence of the crack on the mesoscale mass flow and thus on the macroscopic permeability tensor begins with quite small opening (around $1 \mu \mathrm{m}$ ). 


\subsection{Permeability of RC element under load}

The multiscale framework we present here is suitable for mass transfers within concrete structures or their components. To that aim we focus on experimental results from [8] who designed a coupled tensile - permeability test on Reinforced Concrete $(\mathrm{RC})$ specimens $\left(610 \times 90 \times 90 \mathrm{~mm}^{3}\right.$ concrete element including a $11 \mathrm{~mm}$ diameter reinforcement bar). Here we aim at comparing their experimental measures to numerical results and focus on the permeability evaluation along the failure process.

The fine scale mechanical analysis of this $\mathrm{RC}$ tie is based on a spatial truss representation [12] built with non-adapted meshes. In order to fit to the experimental conditions, displacements are prescribed at both ends of the steel reinforcement bar. Such increasing load leads to progressive cracking of the concrete element and Figure (9) shows a typical crack pattern obtained from a numerical analysis. Three main macroscale cracks are distributed along the tie which is in accordance with experimental observations. Moreover, we show on Figure (9) that there is a quite good agreement between the measured values of those cracks openings during the loading process and the corresponding numerical values.

a)

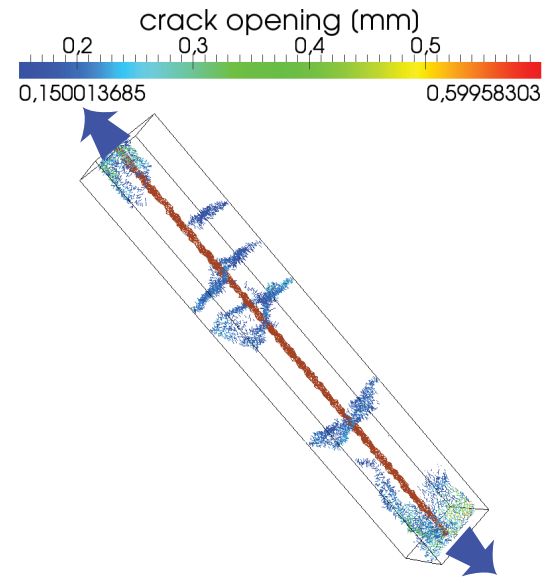

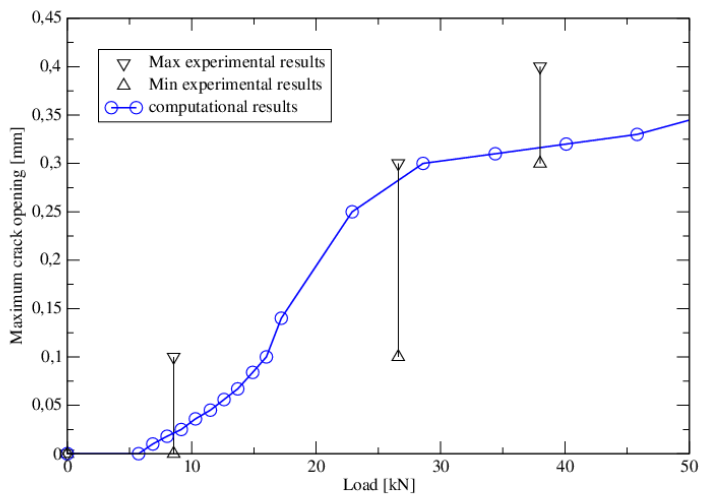

b)

Figure 9: RC tie numerical analysis: a) Numerical crack patterns, b) Maximum crack opening versus load (experimental [8] vs computational results)

On the permeability assessment point of view (here the test deals with water), Figure (10) shows the norm of the mass flow vector at the end of the failure process. In order to match the experiments, this computation has been made imposing mixed boundary conditions: Dirichlet-type along two opposite faces of the specimen and Neumann-type (with zero flow rate) for the four other faces. Although the concrete specimen contains a large number of mesoscale cracks, it is clear that the mass transfer takes place within a subset of those cracks, corresponding to several percolated paths. Thus, on the mass transfer point of view, the former may be seen as a set of macroscale cracks. Hence, it is worth noting that, apart from considering the opening value as the pertinent criterion, this mass transfer analysis leads to an other way to determine this set of macroscale cracks. This analysis being linear and so quite simple to drive, it is a very convenient way to characterize macroscale cracks.

Finally, Figure (11) shows the permeability coefficient - here in $\left[\mathrm{m} \cdot \mathrm{s}^{-1}\right]$ in order to match with the experimental results - evolution along the load increase. The permeability increases from approximatively $2.10^{-10} \mathrm{~m} . \mathrm{s}^{-1}$ to $1.10^{-5} \mathrm{~m} . \mathrm{s}^{-1}$ both for numerical and experimental studies. It is also worth noting that the increasing rate is also quite well represented. Yet, the permeability rise appears for smaller loading values in the numerical study than in the experimental one. Considering the concrete 


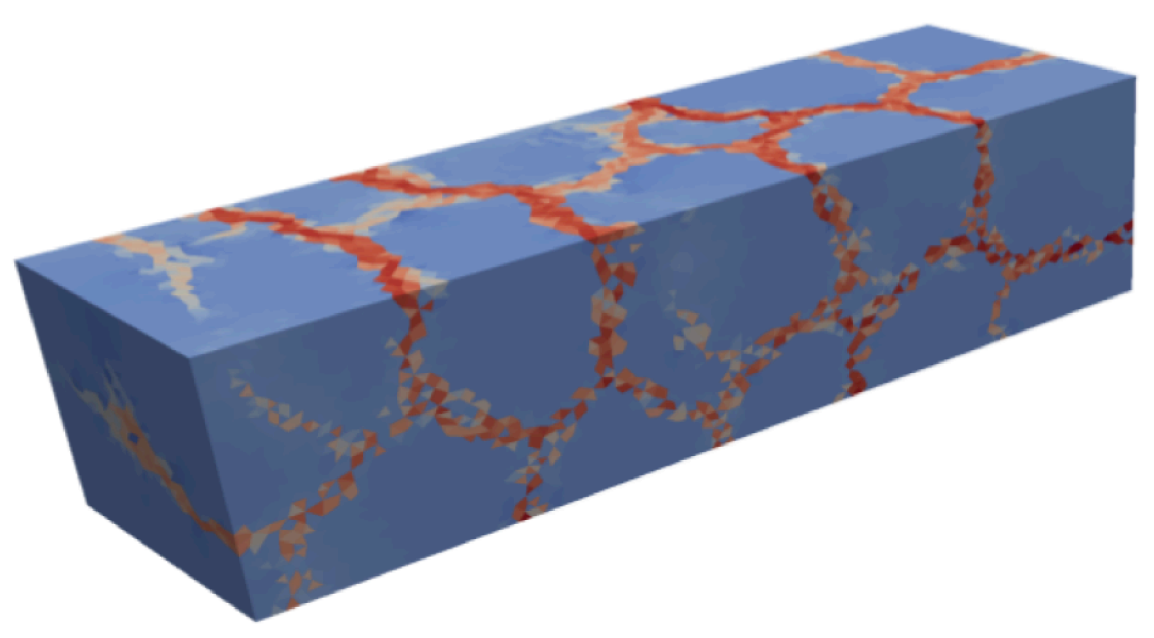

Mass flow
norm $(\mathrm{kg} / \mathrm{s})$
0.000718
0.0001
$1 e-6$
$1 e-8$
$1 e-10$
$1 e-12$
$4.8 e-14$

Figure 10: RC tie numerical analysis: Numerical values of the mass flow vector norm

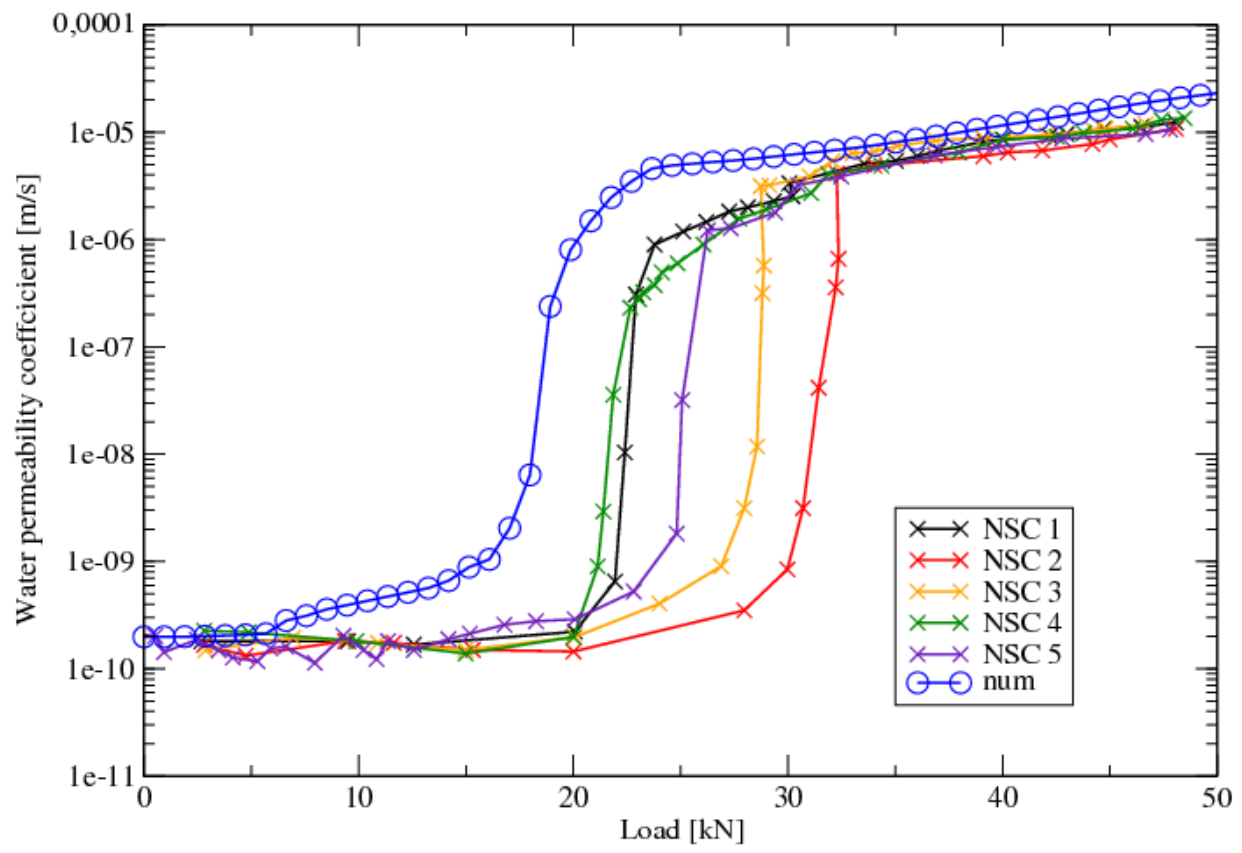

Figure 11: RC tie numerical analysis: Water permeability coefficient versus load [8] vs numerical results

heterogeneity as well as the experimental discrepancy, those results are quite promising. 


\section{Conclusions and outlooks}

In this work we have discussed the use of a sequential two-scales (meso-macro) numerical strategy in order to upscale the permeability of fractured media such as concrete. Sequential multi-scales methods aim at building macroscopic models from a sequence of lower scale computations and require both a macroscale model — here porous media flow — and a fine scale numerical analysis. Such simple upscaling processes made sequential multi-scale methods quite popular in order to compute macroscopic properties of heterogeneous media.

Here the cornerstone relies in the coupling of strong discontinuities — which are supposed to model fine scale fractures within the FE mechanical model - to the fine scale permeability tensor through Poiseuille law. Such coupling is made possible thanks to the strong discontinuity approach which provides the opening value for each of these cracks. Indeed, at such meso level — which we assume to represent heterogeneities from $1 \mathrm{~mm}$ - each crack is a path for an oriented flow and, except for low stress levels, the upscaling process is not possible to manage on a analytical basis. We have presented different numerical tests showing the ability of the numerical upscaling process to model anisotropic macroscopic permeability tensor which confirm the intuitive idea that the whole set of fine cracks cannot lead to an isotropic permeability tensor.

Furthermore, we have compared some experimental results obtained on Reinforced Concrete elements by [8]. Here the heterogeneities are the reinforcement bars and we compute both the mass flow vector and the resultant macroscopic permeability. When compared to the experiments the two-scales numerical analysis shows a good agreement. However, we can observe a high discrepancy within the experimental data. Consequently, further tests dealing with components of RC structures are needed before a complete assessment may be reached.

Finally, it is worth noting that any upscaling process of the permeability may be viewed as an advantageous tool in order to define whether the fracture pattern is percolated (or connected) or not. Actually such feature comes as a by-product results of the two-scales analysis. Hence, to the authors point of view, important efforts should be paid in the near future in order to develop those multi-scale strategies, rather within an integrated framework.

\section{References}

[1] Choinska M. Effets de la température, du chargement mécanique et de leurs interactions sur la perméabilité du béton de structure. PhD Thesis, Ecole Centrale de Nantes \& Université de Nantes 2006.

[2] Picandet V, Khelidj A, Bellegou H. Crack effects on gas and water permeability of concretes. Cement and Concrete Research 2009; 39:537-547. doi=10.1016/j.cemconres.2009.03.009.

[3] Pijaudier-Cabot G, Dufour F, Choinska M. Permeability due to the increase of damage in concrete : From diffuse to localized damage distributions. Journal of Engineering Mechanics 2009; 135:1022-1028. doi=10.1061/(ASCE)EM.1943-7889.0000016.

[4] Shao J, Zhou H, Chau K. Coupling between anisotropic damage and permeability variation in brittle rocks. International Journal for Numerical and Analytical Methods in Geomechanics 2005; 29:1231-1247. doi=10.1002/nag. 457. 
[5] Chatzigeorgiou G, Picandet V, Khelidj A, Pijaudier-Cabot G. Coupling between progressive damage and permeability of concrete: analysis with a discrete model. Int. J. Numer. Anal. Meth. Geomech. 2005; 29:1005-1018. doi=10.1002/nag.445.

[6] Dormieux L, Kondo D. Approche micromécanique du couplage perméabilité - endommagement. C. R. Mecanique 2004; 332:135-140. doi=10.1016/j.crme.2003.11.003.

[7] Feyel F, Chaboche JL. Multi-scale non-linear $\mathrm{FE}^{2}$ analysis of composite structures: damage and fiber size effects. Revue européenne des Éléments Finis: NUMDAM'00 issue 2001; 10:449-472.

[8] Desmettre C, Charron J. Novel water permeability device for reinforced concrete under load. Mat. and Struct. 2011; doi=10.1617/s11527-011-9729-6.

[9] Bear J. Dynamics of Fluids in Porous Media. Dover Publications, 1972.

[10] Hill R. Elastic properties of reinforced solids: some theoretical principles. J. Mech. Phys. Solids 1963; 11:357-372. doi=10.1016/0022-5096(63)90036-X.

[11] Du X, Ostaja-Starzewski M. On the size of representative volume element for darcy law in random media. Proc. R. Soc. 2006; 462:2949-2963. doi=10.1098/rspa.2006.1704.

[12] Benkemoun N, Hautefeuille M, Colliat JB, Ibrahimbegovic A. Failure of heterogeneous materials: 3d meso-scale fe models with embedded discontinuities. International Journal of Numerical Methods in Engineering 2010; 82:1671-1688. doi=10.1002/nme.2816.

[13] Moës N, Cloirec M, Cartraud P, Remacle JF. A computational approach to handle complex microstructure geometries. Computer Methods in Applied Mechanics and Engineering 2003; 192:3163-3177. doi=10.1016/S0045-7825(03)00346-3.

[14] Hillerborg A. Application of the fictious crack model to different types of materials. International Journal of Fracture 1991; 51:95-102. doi=10.1007/BF00033972.

[15] Pijaudier-Cabot G, Bažant ZP. Nonlocal damage theory. Journal of Engineering Mechanics (ASCE) 1987; 113:1512-1533. doi=10.1061/(ASCE)0733-9399.

[16] Wells GN, Sluys LJ. Application of embedded discontinuities for softening solids. Engineering Fracture Mechanics 2000; 65:263-281. doi=10.1016/S0013-7944(99)00120-4.

[17] Brancherie D, Ibrahimbegovic A. Novel isotropic continuum-discrete damage model capable of representing localized failure of massive structures. Part I: theoretical formulation and numerical implementation. Engineering Computation 2009; 26:100-127. doi=10.1108/02644400910924825.

[18] Moës N, Dolbow J, Belytschko T. A finite element method for crack growth without remeshing. International Journal for Numerical Methods in Engineering 1999; 46:131-150. doi=10.1002/(SICI)1097-0207(19990910)46:1.

[19] Benkemoun N, Ibrahimbegovic A, Colliat JB. Anisotropic constitutive model of plasticity capable of accounting for details of meso-structure of two-phase composite material. Computers and Structures 2012; 90-91:153-162. doi=10.1016/j.compstruc.2011.09.003.

[20] Cox D, Isham V. Point Processes. Chapman \& Hall, 1980. 
[21] Brezzi F, Fortin M. Mixed and hybrid finite element methods. Springer-Verlag, 1991.

[22] Kassiotis C, Hautefeuille M. coFeap's Manual 2008. http://www.Imt.ens-cachan.fr/cofeap/ files/cofeap_manual.pdf.

[23] Schewchuk JR. An introduction to the conjugate gradient method without the agonizing pain. Technical Report, School of computer science, Carnagie Mellon University, Pittsburgh, PA 15213 1994. http://www.cs. cmu.edu/〜quake-papers/painless-conjugate-gradient.pdf. 